\title{
COMMUNICATION
}

\section{Transformation maligne d'une hyperplasie verruqueuse exophytique de la langue: à propos d'un cas.}

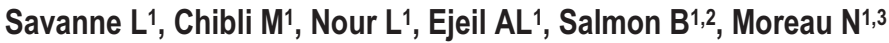

1. Service d'odontologie, Hôpital Bretonneau - Hôpitaux de Paris (AP-HP)

2. Faculté de Chirurgie Dentaire, EA 2496 - Université Paris Descartes, Sorbonne Paris Cité

3. Centre de Psychiatrie et Neurosciences, INSERM UMR 894, Université Paris Descartes - Hôpital Sainte-Anne - Paris

\section{Introduction}

Les lésions verruqueuses de cavité orale regroupent un ensemble de pathologies de nature histologique et d'évolution variées, dont le diagnostic tant clinique qu'histologique est souvent complexe de par l'absence de critères définitifs et de terminologies souvent imprécises.

Compte-tenu des différences d'évolution et donc de prise en charge de ces pathologies, une bonne corrélation clinicopathologique est nécessaire afin de choisir l'option thérapeutique la plus adaptée à l'évolution prévisible de la lésion.

\section{Observation}

Afin d'illustrer l'importance de cette corrélation clinico-pathologique, il est rapporté le cas d'une patiente de 73 ans, en bonne santé générale, non fumeuse, ayant consulté en urgence dans le service d'odontologie de l'hôpital Bretonneau à Paris, pour une « blessure » linguale douloureuse consécutive, d'après la patiente, à une irritation chronique par les cuspides linguales de la 36 fracturée.

L'examen clinique retrouvait une volumineuse masse verruqueuse exophytique blanchâtre, indurée, irrégulière, de $4 \mathrm{~cm}$ de grand axe au niveau du versant latéral et ventro-latéral gauche de la langue mobile. Aucune autre anomalie n'était observée sur le reste de la muqueuse buccale.

Compte-tenu de l'aspect clinique atypique et suspect, il a été pratiqué une biopsie en deux points : un prélèvement au niveau de la zone de plus grande irrégularité morphologique et induration et un prélèvement à cheval entre la masse verruqueuse et la muqueuse linguale saine. L'examen anatomopathologique écartait la possibilité d'un carcinome verruqueux et retrouvait une dysplasie de haut grade (classée OIN 3) associée à un foyer de carcinome épidermoïde micro-invasif.

Au final, il a été posé le diagnostic d'une transformation maligne d'une hyperplasie verruqueuse exophytique.

Après consultation d'annonce, la patiente a été prise en charge dans un service spécialisé en cancérologie (à l'Institut Curie) où elle a bénéficié d'une glossectomie partielle gauche associée à un curage cervical homolatéral. A un an, elle ne présentait aucun signe de récidive locale ou à distance ni de nouvelle lésion suspecte.

\section{Discussion}

L'hyperplasie verruqueuse exophytique est une entité énigmatique, tant sur le plan clinique qu'anatomopathologique, quasi indissociable en clinique du carcinome verruqueux. Ce terme a été proposé en 2013, à la suite de la première conférence de consensus asiatique sur le diagnostic et la terminologie des lésions verruco-papillaires de la cavité orale (Zain et al., 2013) pour définir l'entité clinique correspondant histologiquement à une hyperplasie verruqueuse isolée de la muqueuse orale (Patil et al., 2016), la distinguant d'autres lésions verruqueuses telles que la leucoplasie verruqueuse proliférative ou le carcinome verruqueux.

Dans une étude sur 324 cas d'hyperplasie verruqueuse exophytique, une transformation maligne était observée dans 10 cas : 8 en carcinome épidermoïde et 2 en carcinome verruqueux (Hsue et al., 2007).

\section{Conclusion}

L'hyperplasie verruqueuse exophytique est une lésion verruqueuse peu fréquente de diagnostic clinique et anatomopathologique complexe. Compte-tenu d'un potentiel de transformation maligne, une bonne corrélation clinicopathologique est essentielle à une prise en charge précoce et efficace de cette pathologie.

loicsavanne@club-internet.fr

(C) The authors, published by EDP Sciences. This is an Open Access article distributed under the terms of the Creative Commons Attribution License 4.0 (http://creativecommons.org/licenses/by/4.0/). 